\title{
A young woman with multiple intra cardiac mass, systemic symptoms and stroke
}

\author{
Mohamed Faisal Abdul Hamid \\ Department of Medicine, University of Kebangsaan, Malaysia Medical Centre, Kuala Lumpur Malaysia
}

\begin{abstract}
A 32-year-old female was admitted to hospital following generalised tonic seizure, and recurrent stroke. She had history of young stroke with left hemiparesis at the age of 27 , subsequently defaulted follow up. Evaluation during that time included connective tissue disease and antiphospholipid syndrome screening which were negative and a transthoracic echocardiogram which revealed pericardial effusion of unknown aetiology. A repeat transthoracic and a cardiac computed tomography (CT) scan during this admission revealed multiple intracardiac mass at left and right atrium while CT Brain showed extensive multifocal infarct. Patient also had constitutional symptoms of fever, loss of appetite and loss of weight. A diagnosis of cardiac myxoma was made.The diverse presentation of cardiac myxoma indicates the complexity of the disease. This case illustrates that early diagnosis and treatment of cardiac myxoma is of utmost importance in preventing further sequelae.
\end{abstract}

Key Words: Cardiac myxoma, case report, stroke

\section{Introduction}

Myxomas are the most common primary cardiac tumour. They are neoplasm of endocardial origin. Cardiac myxoma is found more commonly in young adults with stroke or transient ischemic attack (1 in 250) than in older patients with these problems ( 1 in 750) (1). The annual incidence is 0.5 per million population, (2) with $75 \%$ of cases occurring in the left atrium. There is a $2: 1$ female preponderance, (3) and the age at onset is usually between 30 and 60 years.

Clinical presentation varies from obstructive symptoms (54-95\%), embolic phenomenon (10$45 \%)$, and constitutional symptoms (34-90\%) (4). Younger and male patients have more neurologic symptoms, and female patients have more systemic symptoms (2).

This patient has a rare presentation of multicentric cardiac myxoma with both embolic and systemic manifestation. Clinicians should be aware that the presence of embolic phenomena, especially in young patients with neurological symptoms, should prompt early neuroimaging and echocardiography.

\section{Case report}

We report a 32-year-old lady; non-smoker with no known medical illness who had been well until about the age of 27 when she presented with weakness of both the left upper limb and lower limb. Computed tomography (CT) scan of the brain done in another centre showed a right middle cerebral artery (MCA) territory infarct. She was screened for connective tissue disease as well as antiphospholipid syndrome which were negative.

She had residual weakness on the left side of her body, with power of about $4 / 5$, however she was independent on activities of daily living. Subsequently, she defaulted follow up and presented again 5 years later in the same hospital with complex partial seizure of the left upper limb and lower limb. There was no preceding fever, trauma, or headache. She was later transferred to our hospital for further treatment.

Examination revealed blood pressure of $120 / 69 \mathrm{mmHg}$, heart rate of $90-110 /$ minute irregularly irregular, temperature $38^{\circ} \mathrm{C}$, glasgow coma scale (GCS) of 9/15 (E-2, V-2, M-5), pupils $5 \mathrm{~mm}$ bilaterally equal and reactive to light. Neurological examinations were limited by patient's poor GCS. The right upper limb was flaccid, while the rest of the limbs were hypertonic, with positive Hoffman's sign on left side. Reflexes were brisk on the left upper and lower limbs as well as the right lower limb. There were non-sustained clonus bilaterally and babinski response was positive bilaterally. Apart from 
irregularly irregular pulse, cardiovascular examination did not reveal any other abnormalities. Other systems were normal.

Initial investigations revealed white cell count of $13.3 \times 10^{9} / \mathrm{L}$, haemoglobin $11.3 \mathrm{~g} / \mathrm{dL}$ and platelet $100 \times 10^{9} / \mathrm{L}$. Serum electrolytes, liver and renal profile were normal. Blood cultures were negative. Electrocardiogram (ECG) showed normal axis, atrial fibrillation and $\mathrm{T}$ inversion in lead V2-V6. Chest $\mathrm{x}$-ray revealed enlarged left atrium with lobulated mass at the left heart border (Figure 1).

CT Brain (Figure 2) revealed multifocal infarct in left MCA territory and right cerebellum with the presence of cerebral oedema. There were old infarcts in right temporo-parietal and occipital region, right basal ganglia, right head of caudate nucleus, left external capsule and left cerebellum associated with encephalomalacic changes.

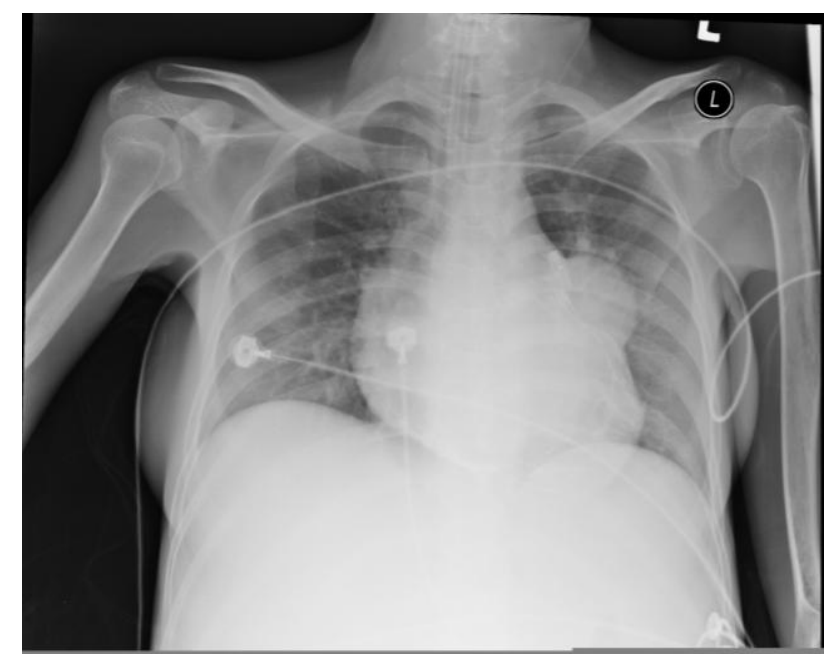

Fig. 1. CXR showed enlarged left atrium with lobulated mass.
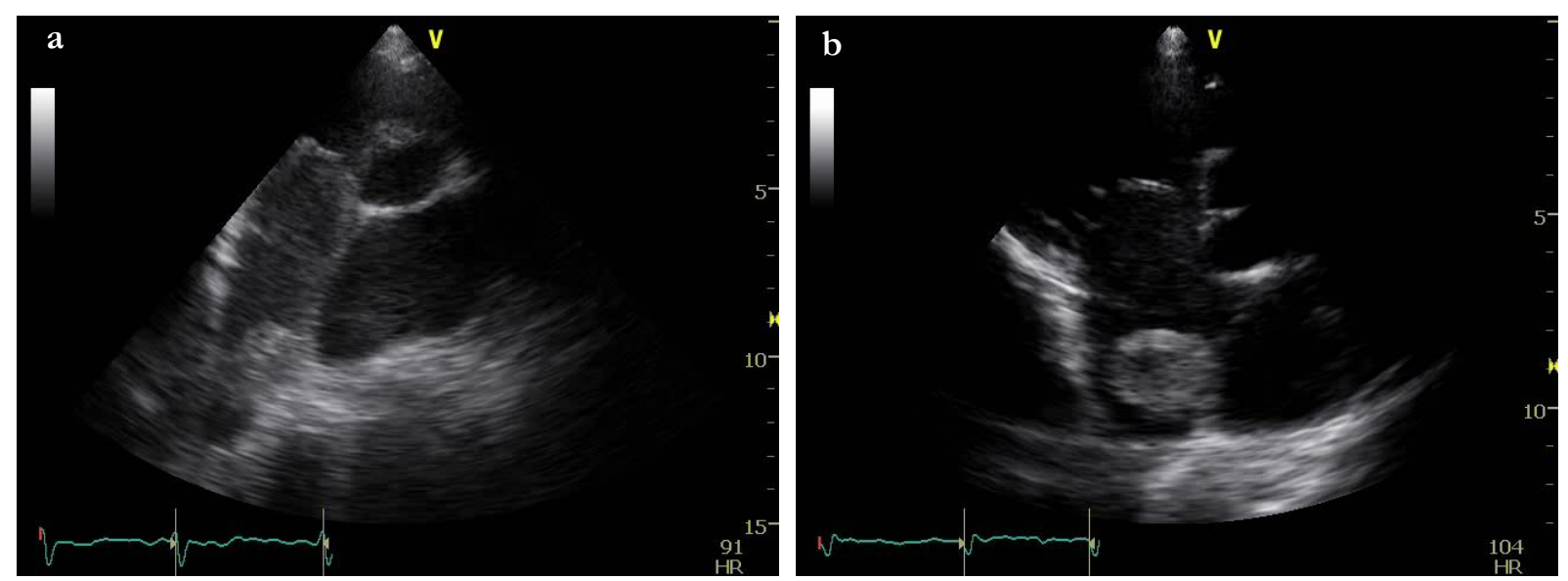

Fig. 3. Transthoracic echocardiogram (4 chamber view) showing right atrial mass (Figure $3 a)$ and left atrial mass (Figure 3b). 


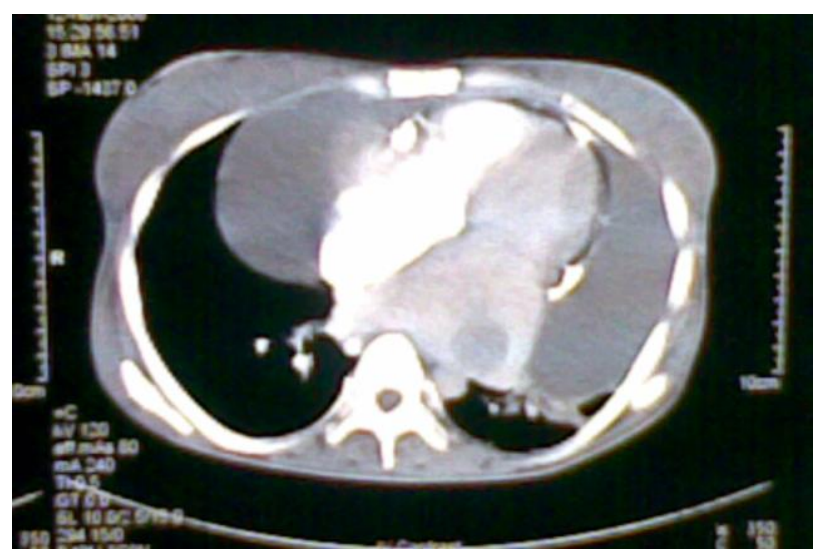

Fig. 4. CT Cardiac showed lobulated mass in the left atrium.

given. Neurosurgical opinion was sought, however there was no intervention by the neurosurgical team. Since patient was generally ill and ventilated, further plan was to wait for neurological recovery before pursuing further management of cardiac myxoma.

Unfortunately patient condition deteriorated further and succumbed to her illness. Cause of death was recurrent extensive CVA with cerebral oedema secondary to atrial fibrillation and atrial myxoma.

\section{Discussion}

Cardiac myxoma is the most common type of primary cardiac tumour, accounting for $1 / 3$ to $1 / 2$ of all cases. $75 \%$ located in the left atrium. About $25 \%$ located in right atrium while small percentages are multi-centric or intraventricular. Most tumours (90\%) arise in the atrial septum in the region of fossa ovale. Our patient has a rare presentation of bilateral atrial myxoma which occurs in less than $5 \%$ of cases.

Although a majority are sporadic, about $7 \%$ are familial, with autosomal dominant inheritance. HSV infection has been reported to be associated with some cases of sporadic atrial myxoma and these may result from chronic inflammation (5).

Familial cases are part of carney complex; autosomal dominant condition characterised by cutaneous spotty pigmentation, cutaneous and cardiac myxomas, nonmyxomatous extra-cardiac tumours and endocrinopathies (6).

Three most common symptoms of cardiac myxoma include dyspnea, palpitations and chest pain. Based on a retrospective study of case notes, ECG and $x$-rays of 171 patients treated for cardiac myxoma from february 1992 to october 2006 which was conducted in south India; (7) embolism was found in $9 \%$ of patients and systemic symptoms in $20 \%$ of patients. Female patients and patients with right atrial myxomas more commonly had systemic symptoms. The same paper states that auscultation findings were present in $89 \%$ of patient, $67 \%$ had mid diastolic murmur while $50 \%$ of patients had tumour plop. In our patients, there were normal heart sound as from the echocardiogram findings, the tumour were located away from the mitral valve and tricuspid valve. Her febrile illness is most likely part of a systemic manifestation of cardiac myxoma.

The patient had extensive multifocal infarct due to emboli from cardiac myxoma. Kinci and Donan et al. (8) reported that based on a review of 113 patients with cardiac myxoma, 93 patients $(82 \%)$ had ischaemic stroke/transient ischaemic attack. Tekin reported a case of a giant atrial myxoma presented as acute stroke and demonstrated that it is necessary to include cardiac tumors in the differential diagnosis of acute stroke (9). Other neurological manifestations (which are not present in our patient) include intracranial haemorrhage, subdural haemorrhage, syncopal attack, psychiatric presentations, headache, seizure, coma, and spinal cord embolism.

Transesophageal echocardiogram (TOE) which has been reported as having 100\% sensitivity for cardiac myxoma, is preferred over transthoracic echocardiography. Cardiac magnetic resonance imaging (MRI) can assist in delineating tumour size, attachment and mobility. This information may be helpful in surgical resection.

Because of the potential life-threatening sequelae of cardiac myxoma, treatment consists of urgent surgical resection even in asymptomatic patients discovered incidentally (4). Surgical excision is considered safe, with an excellent long-term prognosis. Transseptal approach, in allowing total resection of the myxoma, prevents recurrence.

As in our patient, anticoagulant with warfarin is mainly for secondary prevention, since patient had atrial fibrillation. It may not be protective against stroke caused by the cardiac myxoma itself since tumour fragments or adherent thrombus may embolize, with or without anticoagulation. There may be risk of bleeding as the multiple, bilateral fusiform aneurysms commonly found on peripheral arterial branches predispose the patient to cerebral haemorrhage. Therefore, starting patient of cardiac myxoma with warfarin needs thorough assessment of the risk and benefits to the patient. 
As for follow up for patients with sporadic myxoma, annual review with echocardiography is suggested for a period of 3 to 4 years, when the risk of recurrence is greatest. For Carney complex, which has a recurrence rate of up to $25 \%$, lifetime annual review with familial screening is recommended.

Recent study has hypothesised that IL-6 might be used as a marker of myxoma. Cardiac myxomas produce and secrete interleukin 6 (IL-6), which may be a possible explanation for the inflammatory and immune features observed. IL6 level correspond well with tumour size, its level decrease following resection of the tumour and rise again at time of recurrence (10).

This case illustrates the unique presentation of cardiac myxoma. In summary, the presence of embolic phenomena, especially in young patients with neurological symptoms, should prompt early neuroimaging and echocardiography, even in the absence of electrocardiographic or auscultation abnormalities.

\section{References}

1. Hart RG, Albers GW, Koudstaal PJ. Cardioembolic stroke. In: Ginsberg MD, Bogousslavsky J, editors. Cerebrovascular disease: pathophysiology, diagnosis and management. London: Blackwell Science; 1998. p. 1392-1429.

2. Pinede L, Duhaut P, Loire R. Clinical presentation of left atrial myxoma. A series of
112 consecutive cases. Medicine (Baltimore) 2001; 80: 159-172.

3. MacGowan SW, Sidhu P, Aherne T, et al. Atrial myxoma: national incidence, diagnosis and surgical management. Ir J Med Sci 1993; 162: 223-226.

4. O'Rourke F, Dean N, Mouradian MS, Akhtar N, Shuaib A. Atrial myxoma as a cause of stroke: case report and discussion. CMAJ 2003; 169: 1049-1051.

5. Li Y, Pan Z, Ji Y, et al. Herpes simplex virus type 1 infection associated with atrial myxoma. Am J Pathol 2003; 163: 2407-2412.

6. Vidaillet HJ Jr, Seward JB, Fyke FE 3rd, Su WP, Tajik AJ. "Syndrome myxoma": A subset of patients with cardiac myxoma associated with pigmented skin lesions and peripheral and endocrine neoplasms. Br Heart J 1987; 57: 247 255.

7. Aggarwal SK, Barik R, Sarma TC, et al. Clinical presentation and investigation findings in cardiac myxomas: new insights from the developing world. Am Heart J 2007; 154: 1102-1107.

8. Ekinci EI, Donnan GA. Neurological Manifestation of cardiac myxoma: a review of literature and report of cases. Intern Med J 2004; 34: 243-249.

9. Tekin G. Giant Left Atrial Myxoma Presenting with Acute Ischemic Stroke. TJFMPC 2015; 9: 75-77.

10. Cesar EM, Manuel FR, Primo P. Interleukin-6 p and recurrent cardiac myxoma. J Throrac Cardiovasc Surg 2001; 121: 395. 